\title{
Étude botanique, tri phytochimique et évaluation in vitro de l'activité antifongique des extraits de feuilles de Mallotus oppositifolius (Geisel.) Müll. Arg (Euphorbiaceae) sur Fusarium sp. et Phytophthora $s p$. deux champignons phytopathogènes
}

\author{
SARAKA Allou Isidore ${ }^{*}$, ABO Kouabenan ${ }^{2}$, OUATTARA Katinan Etienne ${ }^{1}$ et ZIRIHI \\ Guédé Noël ${ }^{1}$ \\ 1*Laboratoire de Botanique, UFR Biosciences, Université Félix Houphouët Boigny de Cocody Abidjan; 22 BP 582 Abidjan \\ 22 \\ 2 Laboratoire de Phytopathologie et de Biologie Végétale, Institut National Polytechnique Félix Houphouët-Boigny (INP-HB), \\ BP 1313 Yamoussoukro, Côte d'Ivoire \\ Auteur correspondant, email: asaraka01@yahoo.fr / cel: (+225) 07790483
}

Mots clés : Screening phytochimique, Activité antifongique, Mallotus oppositifolius, District de Yamoussoukro

Keywords: Phytochemical Screening, Antifungal activity, Mallotus oppositifolius, District of Yamoussoukro.

Publication date 31/08/2019, http://www.m.elewa.org/JAPS

\section{RESUME}

L'objectif de ce travail est d'évaluer le pouvoir fongicide des feuilles de Mallotus oppositifolius sur Phytophthora sp. et Fusarium sp. deux champignons phytopathogènes dans le cadre de la recherche de substances naturelles d'origine végétale comme alternative aux pesticides chimiques en agriculture. Une enquête ethnobotanique réalisée auprès de quarante tradipraticiens de santé du District de Yamoussoukro sur les Euphorbiaceae médicinales a permis d'identifier treize espèces. L'étude a montré que les maladies de la peau $(36 \%)$ étaient majoritairement traitées par ces Euphorbiaceae dont Mallotus oppositifolius. Au vue de ces données et celles de la littérature, Mallotus oppositifolius a été retenu pour l'évaluation du potentiel antifongique contre Fusarium sp. et Phytophthora sp. L'activité antifongique a été évaluée par la méthode de double dilution sur milieu PDA avec des extraits éthanoliques et aqueux dérivés des feuilles de cette plante. Les résultats ont montré que les extraits aqueux et éthanoliques $70 \%$ de Mallotus oppositifolius possèdent des propriétés antifongiques à la fois sur Fusarium sp. et Phytophthora sp. Les activités antifongiques ont été plus importantes avec les extraits éthanoliques qu'avec les extraits aqueux. Avec une concentration minimal inhibitrice CMI = $25 \pm 0,0 \mathrm{mg} / \mathrm{mL}$, une $\mathrm{CI}_{50}=6,5 \pm 0,05 \mathrm{mg} / \mathrm{mL}$ et une concentration minimal fongicide $\mathrm{CMF}=$ $200 \pm 0,0 \mathrm{mg} / \mathrm{mL}$ Fusarium sp. semble être le plus sensible. Phytophthora sp. est moins sensible avec une $\mathrm{CMI}=50 \pm 0,0 \mathrm{mg} / \mathrm{mL}$, une $\mathrm{CI}_{50}=12 \pm 0,05 \mathrm{mg} / \mathrm{mL}$ et une $\mathrm{CMF}=200 \pm 0,0 \mathrm{mg} / \mathrm{mL}$. Le screening phytochimique a mis en évidence la présence de sept groupes de composés parmi lesquels les alcaloïdes, les polyterpènes et stérols, les tanins galliques et les catéchines sont les plus abondants dans les extraits éthanoliques. En plus de ces composés, les flavonoïdes, les polyphénols ainsi que les saponines sont aussi bien présents dans les extraits aqueux que dans les extraits alcooliques de Mallotus oppositifolius. Ces composés pourraient justifier l'activité des extraits éthanoliques de Mallotus oppositifolius. Ces résultats peuvent servir pour les recherches agronomiques dans le cadre de la lutte contre les deux champignons phytopathogènes étudiés. 
Botanical study, phytochemical screening and in vitro evaluation of the antifungal activity of Mallotus oppositifolius (Geisel.) Müll. Arg. (Euphorbiaceae) leaf extracts on Fusarium sp. and Phytophthora sp., two phytopathogenic fungi.

\begin{abstract}
The purpose of this work is to evaluate the fungicidal power of the leaves of Mallotus oppositifolius on Phytophthora sp. and Fusarium sp. two phytopathogenic fungi in the research of natural substances of plant origin as an alternative to chemical pesticides in agriculture.

An ethnobotanical survey of forty traditional healers in the Yamoussoukro District on medicinal Euphorbiaceae identified thirteen species. The study showed that skin diseases (36\%) were mainly treated by these Euphorbiaceae including Mallotus oppositifolius. In view of these data and those in the literature, Mallotus oppositifolius was selected for the evaluation of the antifungal potential against Fusarium sp. and Phytophthora sp. The antifungal activity was evaluated by the double dilution method on PDA medium with ethanolic and aqueous extracts derived from the leaves of this plant. The results showed that aqueous and ethanolic extracts $70 \%$ of Mallotus oppositifolius have antifungal properties on both Fusarium sp. and Phytophthora sp. Antifungal activities were more important with ethanolic extracts than with aqueous extracts. With a minimum inhibitory concentration MIC = $25 \pm 0.0 \mathrm{mg} / \mathrm{mL}$, a minimal concentration for $50 \%$ of inhibition $\mathrm{IC}_{50}=6.5 \pm 0.05 \mathrm{mg} / \mathrm{mL}$ and a minimum fungicide concentration MFC $=200 \pm 0.0 \mathrm{mg} / \mathrm{mL}$ Fusarium sp. appears to be the most sensitive. Phytophthora sp. is less sensitive with an MIC $=50 \pm 0.0 \mathrm{mg} / \mathrm{mL}$, an IC $50=12 \pm 0.05 \mathrm{mg} / \mathrm{mL}$ and a MFC $=200 \pm 0.0 \mathrm{mg} / \mathrm{mL}$. Phytochemical screening revealed the presence of seven groups of compounds among which alkaloids, polyterpenes and sterols, gallic tannins and catechins are the most abundant in ethanol extracts. In addition to these compounds, flavonoids, polyphenols and saponins are present in both aqueous and alcoholic extracts of Mallotus oppositifolius. These compounds could justify the activity of the ethanolic extracts of Mallotus oppositifolius.

These results can be used for agronomic research to control the two phytopathogenic fungi studied.
\end{abstract}

\section{INTRODUCTION}

La filière agricole, malgré son importance et les opportunités qu'elle offre pour l'économie mondiale, fait face à de nombreuses contraintes dont la pression parasitaire qui peut réduire les rendements de 90\% (Coraf, 2010). Ces parasites constituent une contrainte biotique majeure tant pour la production que pour les perspectives futures d'intensification de celle-ci. $\mathrm{Au}$ nombre de ces parasites, figurent les champignons phytopathogènes tels que les espèces des genres Fusarium et Phytophthora. Les espèces du genre Fusarium s'attaquent aux cultures telles que la tomate (Hibar et al., 2007), le cotonnier et le bananier (Soro et al., 2012) ainsi que le riz (Zehharb et al., 2006). Des études effectuées en 2015 dans trois zones de production de banane plantain en Côte d'Ivoire (à Anyama et à la périphérie d'Abidjan) ont mis en évidence la présence des foyers de fusariose et dont l'agent causal était Fusarium oxysporum (Kra et al., 2011). Par ailleurs Phytophthora sp., agent causal de la pourriture brune, crée de nombreux dégâts chaque année, allant jusqu'à la perte de la totalité de la production de cacao dans certains ays (Flood, 2006). Au niveau mondial, cette maladie engendre des pertes de production de l'ordre de $30 \%$ (Lass, 1985). Face à ces contraintes, différentes stratégies de lutte ont été développées afin de réduire les dégâts dus à ces maladies. Au nombre de cellesci, figure la méthode chimique basée sur l'utilisation de produits de synthèse. Cependant, l'utilisation de ces produits chimiques bien qu'efficace constitue une menace pour la santé humaine, animale et environnementale (Picanc et al., 1998). De plus, leur utilisation répétée peut entrainer l'apparition de phénomènes de résistance chez les bioagresseurs (Brent et 
Hollomon, 2007. Au regard de cela, des recherches sont orientées vers les substances naturelles comme alternative. La tendance actuelle est la formulation de biofongicides à partir de substances naturelles d'origine végétale. Ces substances ont l'avantage d'être biodégradables et non rémanentes dans la nature. C'est dans ce contexte qu'une étude ethnobotanique a été conduite dans le District de Yamoussoukro, en Côte d'Ivoire.Elle a permis d'identifier 13 Euphorbiaceae médicinales utilisées par les autochtones Baoulé, parmi lesquelles Mallotus oppositifolius (Geisel.) Müll. Arg. C'est une plante qui intervient en médecine traditionnelle ivoirienne dans les soins de plusieurs pathologies : la diarrhée, la toux, les infections urinaires, la tuberculose, les plaies chroniques, l'ulcère de Burili (Soladoya et al., 2013, Kouadio et al., 2015). Une étude réalisée sur deux souches phytopathogènes de Sclerotium rolfsii a révélé le pouvoir antifongique des extraits aqueux et hydro-alcooliques des feuilles de cette plante (Orsot et al., 2015). C'est un arbuste pouvant atteindre 4 à $6 \mathrm{~m}$ de haut. Les feuilles inégales, opposées, lancéolées portent quelques poils étoilés. Le limbe est ovale, triangulaire, largement arrondi à la base et mesure 8 à $15 \mathrm{~cm}$ de long et 6 à $10 \mathrm{~cm}$ de large, avec un sommet en pointe acuminée et porte à sa base trois nervures. Le bord du limbe est denté et sa face inférieure est criblée de petites lenticelles dorées, très fines. Le pétiole est long de 1 à 5 $\mathrm{cm}$. L'inflorescence, axillaire, spiciforme, portant de petites fleurs blanchâtres, est un racème long de 5 à $10 \mathrm{~cm}$. Les fruits sont des capsules trilobées, larges de 7 à $8 \mathrm{~mm}$ et formées de 3 loges accolées. Cette espèce est répandue en Afrique intertropicale dans les formations anthropiques. Elle est présente dans toutes les forêts denses et semi-décidues, en Côte d'Ivoire (Anonyme, 2018). Le but de ce travail est d'évaluer le pouvoir fongicide des feuilles de Mallotus oppositifolius sur deux champignons phytopathogènes économiquement importants que sont Phytophthora sp. et Fusarium sp.

\section{MATERIEL ET METHODES D'ETUDE}

\subsection{Materiel}

3.1.1 Matériel végétal: Le matériel végétal est constitué de feuilles de Mallotus oppositifolius (Geisel.) Müll. Arg. (Figure 1). Elles ont été récoltées à Seman un village situé à $8 \mathrm{Km}$ environ de Yamoussoukro. Ces feuilles nt été utilisées pour le tri phytochimique et l'évaluation de l'activité antifongique.

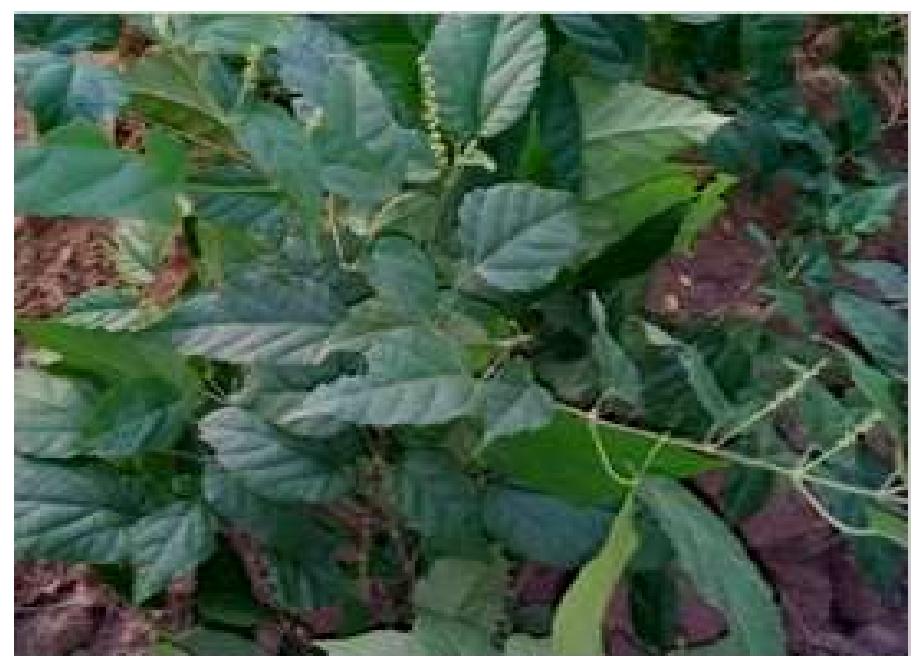

Figure 1: Rameau feuillé, fleuri de Mallotus oppositifolius Source photo SARAKA (Août 2017) 
3.1.2 Matériel fongique: Le matériel fongique est constitué d'isolats de Phytophthora $s p$. et de Fusarium sp. Ces isolats ont été fournis par le Laboratoire de Phytopathologie et de Biologie Végétale de l'Institut National Polytechnique Félix Houphouët-Boigny de Yamoussoukro (Côte d'Ivoire).

\subsection{METHODES}

3.2.1 Enquête ethnobotanique : L'enquête ethnobotanique a été réalisée dans le District de Yamoussoukro, auprès de quarante guérisseurs répartis dans quatre sous-préfectures et vingt villages.

3.2.2 Récolte et séchage des organes végétaux : Les feuilles de Mallotus oppositifolius ont été récoltées en août 2017, à la suite de l'enquête ethnobotanique. Elles ont été lavées à l'eau de robinet, séchées dans une salle à la température ambiante $\left(26^{\circ} \mathrm{C}\right)$ et à l'abri du soleil pendant 21 jours.

3.2.3 Préparation des extraits aqueux et éthanolique: Les feuilles séchées ont été broyées finement grâce à une broyeuse électrique de type Grinder GLG-450. La fine poudre obtenue a subi une extraction selon la méthode de Zirihi et al. (2003). Ainsi, cent grammes (100) g de poudre d'organe végétal ont été mélangés, dans un litre (1L) d'eau distillée grâce à un mixeur de type Blender, trois fois pendant trois minutes. L'homogénat obtenu est d'abord essoré dans un carré de tissu pour éliminer les débris végétaux puis filtré successivement deux fois sur du coton hydrophile pour éliminer les débris qui ont pu traverser les mailles du tissu. Enfin, une dernière filtration est faite une fois sur du papier filtre Wattman. Le filtrat obtenu est séché à l'étuve à $50^{\circ} \mathrm{C}$ pendant cinq jours. La poudre ainsi obtenue est l'extrait total aqueux noté $\mathrm{ETA}_{14}$. Il a été mis dans un récipient stérile puis fermé hermétiquement et conservé au réfrigérateur à $6^{\circ} \mathrm{C}$ jusqu'à utilisation. L'extrait éthanolique a été obtenu selon la deuxième variante de la méthode de Zirihi et al. (2003). Cette extraction est faite de manière similaire à celle de l'extrait aqueux, mais avec l'éthanol $70 \%$ comme solvant. L'extrait éthanolique, codé $\mathrm{EE}_{14}$ a été conservé dans les mêmes conditions que l'extrait aqueux. Ces deux extraits ont été pesés pour l'évaluation du rendement de l'extraction.

3.2.4 Calcul du rendement : Le rendement est déterminé par le rapport de la masse de l'extrait sec après évaporation sur la masse de la poudre de matière végétale sèche utilisée pour l'extraction. Il est exprimé en pourcentage et est calculé selon la formule suivante :

$\mathbf{R d}=(\mathrm{m} \times 100) / \mathbf{M}$;

Où Rd désigne le rendement d'extraction en pourcentage, $\mathbf{m}$, la masse en gramme de l'extrait sec et, $\mathbf{M}$ la masse en gramme de la poudre d'organe végétal.

Le rendement est d'autant important qu'il permet de prévoir la quantité de matière active exploitable à partir d'une méthode d'extraction donnée. Il permet également de prévoir la quantité de matière sèche active exploitable d'un organe donné issu d'une même plante ou de plantes différentes.

3.2.5 Screening phytochimique : Quelques groupes chimiques ont été recherchés dans les extraits (aqueux et hydro-alcoolique) de Mallotus oppositifolius suivant les méthodes classiques de caractérisation par réactions colorées (Mangambu et al., 2014). Les solutions présentant des réactions positives indiquent la présence des groupes chimiques mis en évidence dans l'extrait. Les résumés des réactions sont contenus dans le Tableau 1. 
Tableau 1 : Réactions d'identification des groupes chimiques et indicateurs utilisés

\begin{tabular}{|c|c|c|c|}
\hline \multicolumn{2}{|c|}{ Groupes chimiques } & Réactifs d'identification & Indicateur (Réaction positive) \\
\hline \multicolumn{2}{|c|}{ Stérols et polyterpènes } & $\begin{array}{l}\text { Anhydride acétique, Acide } \\
\text { sulfurique concentré }\end{array}$ & $\begin{array}{l}\text { Apparition à l'interphase d'un anneau } \\
\text { pourpre ou violet, virant au bleu puis au } \\
\text { vert. }\end{array}$ \\
\hline \multicolumn{2}{|c|}{ Polyphénols } & Chlorure ferrique $\mathrm{FeCl} 3(2 \%)$ & $\begin{array}{l}\text { Apparition d'une coloration bleue noirâtre } \\
\text { ou verte plus ou moins foncée }\end{array}$ \\
\hline \multicolumn{2}{|c|}{ Flavonoïdes } & $\begin{array}{l}\text { Alcool chlorhydrique, Copaux } \\
\text { de Magnésium, Alcool iso- } \\
\text { amylique }\end{array}$ & $\begin{array}{l}\text { Dégagement de chaleur puis coloration } \\
\text { rose-orange ou violacée }\end{array}$ \\
\hline \multirow{2}{*}{ Tanins } & Catéchiques & $\begin{array}{l}\text { Formaldéhyde, Acide } \\
\text { chlorhydrique concentré }\end{array}$ & Précipité gélatineux (en gros flocons) \\
\hline & Galliques & $\begin{array}{l}\text { Acétate de sodium, Chlorure } \\
\text { ferrique }\end{array}$ & Coloration bleue-noire intense \\
\hline \multicolumn{2}{|c|}{ Quinones } & Ammoniaque & $\begin{array}{l}\text { Apparition d'une coloration allant du } \\
\text { rouge au violet. }\end{array}$ \\
\hline \multicolumn{2}{|c|}{ Saponosines } & Indice de mousse & Apparition d'une mousse persistante \\
\hline \multirow{2}{*}{\multicolumn{2}{|c|}{ Alcalö̈des }} & $\begin{array}{l}\text { Dragendorff (Solution iodo- } \\
\text { bismuthate de potassium) }\end{array}$ & \multirow{2}{*}{ Précipité de coloration brun rougeâtre } \\
\hline & & $\begin{array}{l}\text { Burchard (Reaction iodo- } \\
\text { iodurée) }\end{array}$ & \\
\hline
\end{tabular}

\subsubsection{Evaluation de l'activité} antifongique: L'évaluation de l'activité antifongique a été réalisée sur des isolats de Phytophthora sp. et de Fusarium sp. Le milieu PDA (Potato Dextrose Agar) a été utilisé pour la culture des champignons. Une gamme de cinq concentrations a été retenue pour chaque extrait $\left(\mathrm{ETA}_{14}\right.$ et $\left.\mathrm{EE}_{14}\right)$. Ces concentrations sont: $100 \mathrm{mg} / \mathrm{mL}, 50 \mathrm{mg} / \mathrm{mL}, 25 \mathrm{mg} / \mathrm{mL}, 12,5$ $\mathrm{mg} / \mathrm{mL}$ et $6,25 \mathrm{mg} / \mathrm{mL}$. Elles ont été définies selon la méthode de la double dilution de liaison géométrique de raison $1 / 2$ (Zirihi et al., 2003). Les différentes quantités des extraits végétaux ont été incorporées aux milieux de culture dans des bouteilles de $500 \mathrm{~mL}$ SCHOTT DURAN. Ces milieux ont été stérilisés à l'autoclave à $120^{\circ} \mathrm{C}$ pendant 20 minutes. Les témoins n'ont reçu aucun apport d'extraits. Après homogénéisation, les différents mélanges, en surfusion à $40{ }^{\circ} \mathrm{C}$, ont été coulés dans des boîtes de Pétri de $90 \mathrm{~mm}$ de diamètre, sous une hotte dans des conditions aseptiques autour d'un bec BUNSEN. Les essais ont été répétés trois fois pour chaque concentration et pour chaque isolat fongique. Les boites de Pétri ont été maintenues sous la hotte jusqu'à la solidification du milieu et à l'ensemencement du fragment mycélien. Un explant de $6 \mathrm{~mm}$ de diamètre de chaque isolat fongique a été prélevé au niveau du front de croissance du champignon dans la boîte de culture. L'explant a été placé au centre de la boîte de Pétri sur le milieu solidifié. Les boîtes de Pétri ensemencées ont ensuite été scellées avec du film adhésif et mises en incubation à l'étuve pendant toute la durée des mesures à 27 $\pm 2^{\circ} \mathrm{C}$. La mesure de la croissance radiale moyenne du mycélium des différents isolats fongiques a été effectuée quotidiennement parallèlement à celle du témoin. Cette mesure s'est réalisée en millimètre en suivant deux axes perpendiculaires tracées au revers de la boîte de Pétri. Les diamètres du mycélium ont été mesurés jusqu'à ce que dans les lots témoins, les 
colonies mycéliennes atteignent la périphérie de la boîte de Pétri. C'est au septième jour post ensemencement que la prise de mesure a été arrêtée. Le taux d'inhibition de la croissance radiale mycélienne a été calculé selon la formule de Leroux et Credet (1978).

\section{$T(\%)=(D-d) / D) \times 100$}

( $\mathbf{T}$ : taux d'inhibition, $\mathbf{D}$ : croissance mycélienne dans les boîtes de Pétri témoins, $\mathbf{d}$ : croissance mycélienne dans les boîtes essais).

La détermination du taux d'inhibition de la croissance mycélienne de chaque souche fongique a permis de définir, pour chaque extrait, la concentration minimale inhibitrice (CMI) et la concentration minimale fongicide (CMF). La CMI correspond à la concentration qui donne $100 \%$ d'inhibition et la CMF correspond à la plus petite concentration à partir de laquelle aucune croissance mycélienne n'est observée dans les nouvelles boîtes de Pétri

\section{RÉSULTATS}

4.1 Rendement des extraits : Le rendement varie en fonction de l'espèce végétale, de l'organe utilisé, des conditions de séchage, du contenu de chaque espèce végétale en métabolites et de la nature du solvant utilisé dans l'extraction. A partir de $100 \mathrm{~g}$ de poudre de feuilles de Mallotus oppositifolius nous avons obtenu 26,35 $\pm 0,75 \mathrm{~g}$ de poudre d'extrait sec par l'extraction aqueuse et $34,47 \pm 1,25 \mathrm{~g}$ par l'extraction hydro-éthanolique. où les explants ont été réensemencées après avoir déterminé la CMI. La concentration qui donne $50 \%$ du taux d'inhibition de la croissance des colonies fongiques $\left(\mathrm{CI}_{50}\right)$ a été déterminée graphiquement (Ackah, 2004).

3.2.7 Traitement et analyse statistique des données : Les moyennes des taux d'inhibitions ont été calculées pour chaque concentration d'extrait relativement à chaque isolat fongique au terme des sept jours d'incubation. Les calculs numériques et la construction des graphiques ont été effectués avec les logiciels XLSTAT 2019 et Excel 2010. Le logiciel XLSTAT a servi à l'analyse de la variance à un facteur (ANOVA 1). Il a été utilisé pour comparer les moyennes des taux d'inhibition des différents extraits sur chaque isolat fongique et aussi les moyennes des taux d'inhibition entre les deux isolats, au seuil de probabilité $p$ inférieure à $5 \%$. Les moyennes ont été classées selon le test LSD de Fisher.

\subsection{Screening phytochimique: Le} screening phytochimique de l'extrait aqueux des feuilles de Mallotus oppositifolius a mis en évidence la présence de sept groupes de composés chimiques horsmis les quinones. Par contre tous les groupes testés ont été mis en évidence dans l'extrait éthanolique (Tableau 2).

Tableau 2 : Métabolites secondaires identifiés dans les extraits (aqueux et éthanolique)

\begin{tabular}{l|l|l|l|l|l|l|l|l}
\hline \multicolumn{2}{l}{ Groupes chimiques } \\
\hline \multicolumn{2}{l|}{ Sapo } & Polyphén & Flavo & $\begin{array}{l}\text { Polyter et } \\
\text { stérol }\end{array}$ & Alca & $\begin{array}{l}\text { Tanin } \\
\text { gall }\end{array}$ & $\begin{array}{l}\text { Tanin } \\
\text { catéch }\end{array}$ & Quin \\
\hline ETA M.o & + & + & + & ++ & ++ & ++ & ++ & - \\
\hline EE M.o & + & + & + & ++ & ++ & ++ & ++ & + \\
\hline
\end{tabular}

M. o. : Mallotus oppositifolius ; - : Absent, + : Présent ; ++ : Abondant.

ETA : Extrait total aqueux ; EE : Extrait éthanolique $70 \%$

Sapo.: Saponines, Polyphén. : Polyphénols, Flavo.: Flavonoïdes, Polyter et stérol.: Polyterpène et stérol, Alca.: Alcaloïdes,Tanin gall. : Tanin gallique, Tanin catéch. : Tanin catéchique, Quin. : Quinones. 


\subsection{Taux d'inhibition de la croissance} mycélienne : Les observations de l'effet des extraits aqueux sur la croissance mycélienne des isolats de Phytophthora sp. et de Fusarium sp. ont révélé, comparativement au témoin, une diminution de la croissance mycélienne au fur

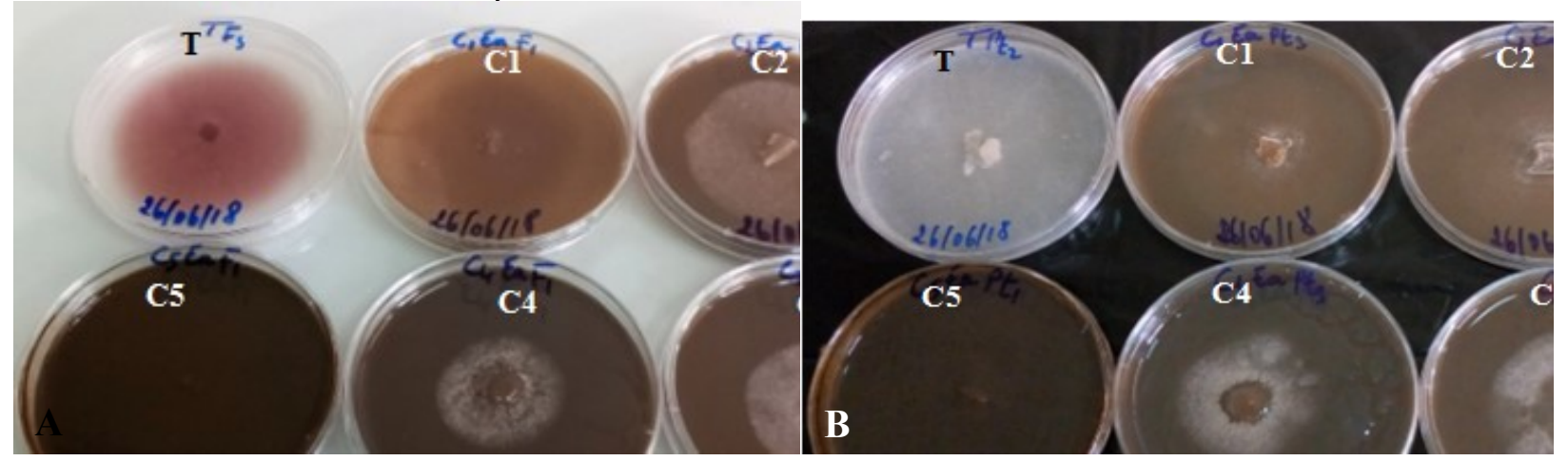

Figure 2 : Croissance in vitro du mycélium de Fusarium sp. et Phytophthora sp. en présence d'extrait aqueux de feuilles de Mallotus oppositifolius (ETA $\left.{ }_{14}\right)$ après 7 jours d'incubation

A : Fusarium sp. B : Phytophthora sp.

Témoin (T) : 0 mg/mL; C1 : 6,25 mg/mL; C2 : 12,5 mg/mL; C3 : 25 mg/mL; C4 : 50 mg/mL; C5 : 100 mg/mL

Avec l'extrait aqueux, le plus fort taux d'inhibition de $100 \%$ a été obtenu avec la concentration de $100 \mathrm{mg} / \mathrm{mL}$ aussi bien sur les isolats de Phytophthora sp. que ceux de Fusarium $s p$. (Figure 3). Les taux d'inhibitions obtenus sont significativement différents d'une concentration à une autre pour chaque colonie fongique. A concentration égale, il n'y a pas de différence significative entre les taux d'inhibition obtenues sur Fusarium sp. et et à mesure que les concentrations des extraits ETA $_{14}$ augmentent dans les boîtes de pétri (Figure 2). Le mycélium atteint la périphérie de la boîte dans les témoins le 7ème jour d'incubation. 


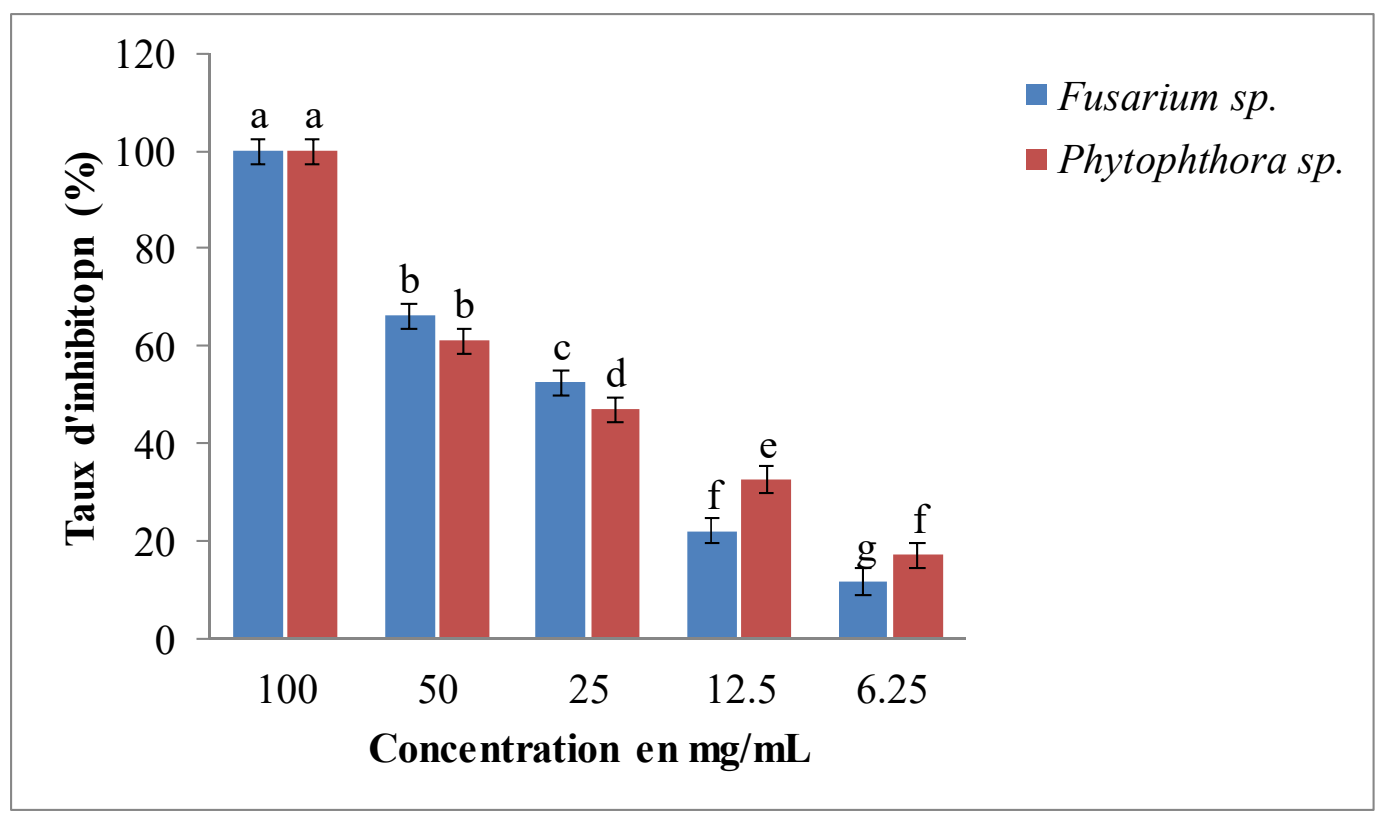

Figure 3: Taux d'inhibition de la croissance mycélienne de Fusarium sp. et Phytophthora sp. en fonction de la concentration d'ETA 14

La Figure 4 présente une diminution de la croissance mycélienne des isolats de Phytophthora $s p$. et de Fusarium sp. au fur et à mesure que les concentrations des extraits $\mathrm{EE}_{14}$ augmentent dans les boîtes de pétri. Le mycélium atteint la périphérie de la boîte dans les témoins le 7ème jour d'incubation.

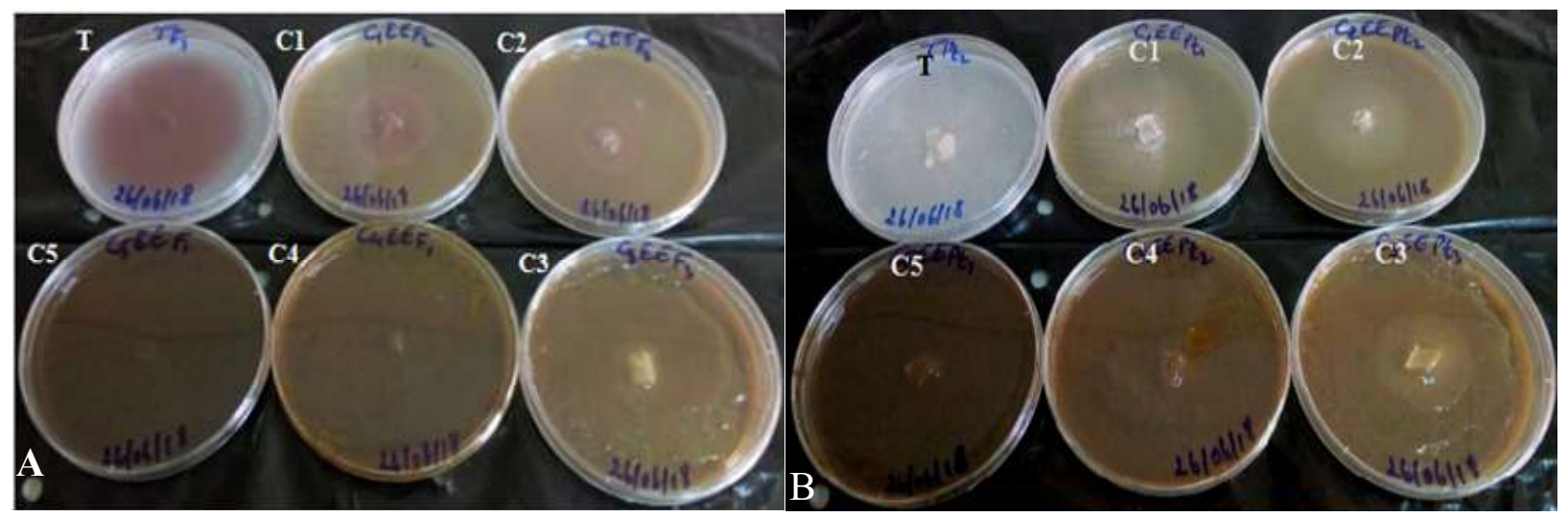

Figure 4 : Croissance in vitro du mycélium de Fusarium sp. et Phytophthora sp. en présence d'extrait éthanolique de feuilles de Mallotus oppositifolius $\left(\mathrm{EE}_{14}\right)$ après 7 jours d'incubation A : Fusarium sp.; B : Phytophthora sp.

Témoin (T): 0 mg/mL; C1: 6,25 mg/mL; C2: 12,5 mg/mL; C3: $25 \mathrm{mg} / \mathrm{mL} ; C 4: 50 \mathrm{mg} / \mathrm{mL} ;$ C5: $100 \mathrm{mg} / \mathrm{mL}$.

Avec l'extrait éthanolique, aucune croissance mycélienne n'a été observée chez Fusarium sp. à partir de la concentration de $25 \mathrm{mg} / \mathrm{mL}$ et aucune croissance n'a été observée chez Phytophthora sp. à partir de la concentration de
$50 \mathrm{mg} / \mathrm{mL}$ (Figure 5). Les taux d'inhibitions obtenus sont significativement différents d'une concentration à une autre aussi bien pour les isolats de Phytophthora sp. que ceux de Fusarium $s p$. A concentration égale, il n'y a pas de 
différence significative entre les taux d'inhibition obtenues sur les deux colonies fongiques (Fusarium sp. et Phytophthora sp.) à partir de $25 \mathrm{mg} / \mathrm{mL}$. Cependant, les taux d'inhibitions obtenus sur les deux colonies fongiques sont significativement différents pour les concentrations $\mathrm{C} \leq 12,5 \mathrm{mg} / \mathrm{mL}$. A ces concentrations les taux obtenus sur Fusarium sp. sont significativement supérieurs à ceux obtenus sur Phytophthora sp.

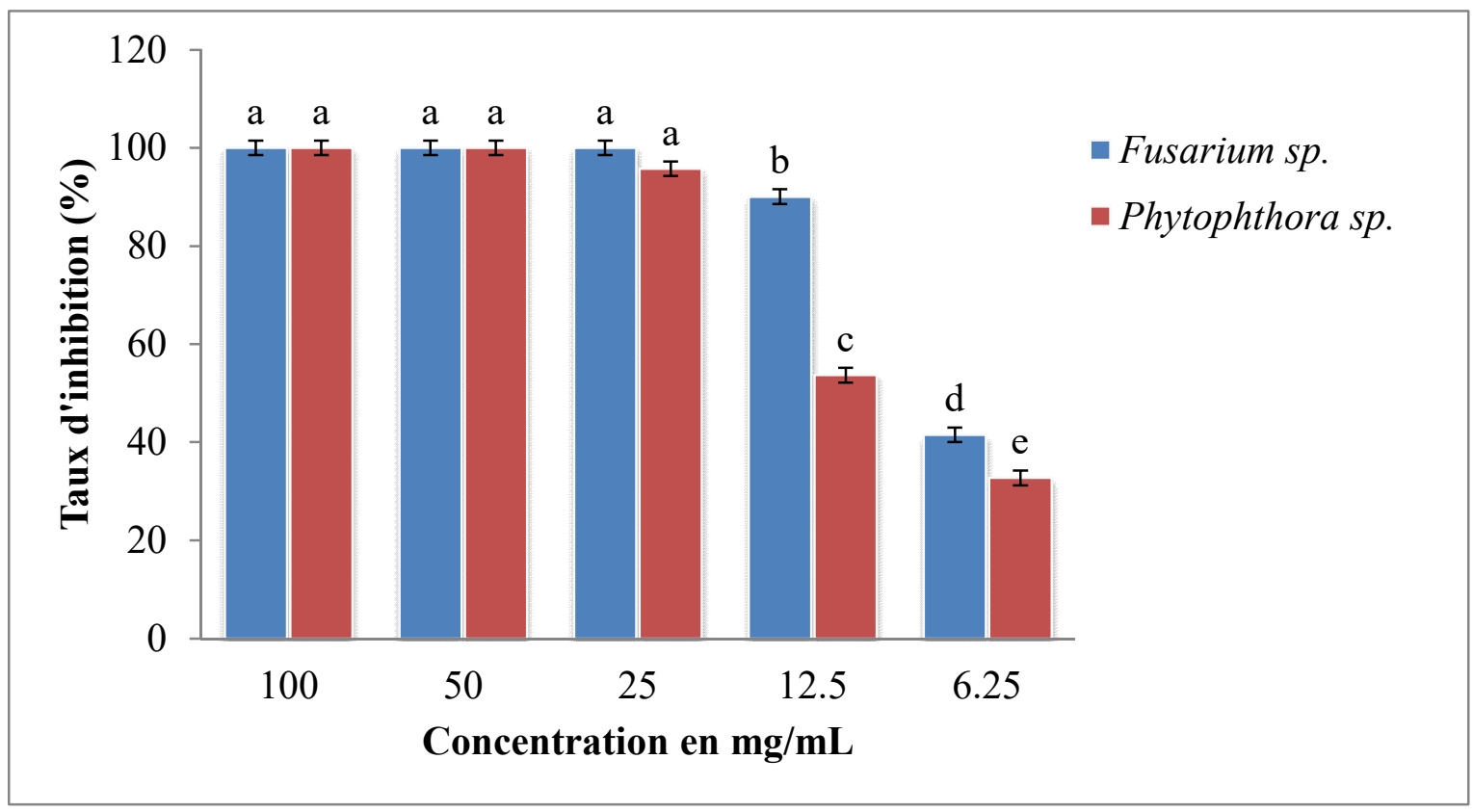

Figure 5 : Taux d'inhibition de la croissance mycélienne de Fusarium sp. et Phytophthora sp. en fonction de la concentration d'EE $\mathrm{E}_{14}$

\subsection{Concentration minimales inhibitrice} (CMI) et fongicide (CMF) : La recherche des concentrations minimales inhibitrice (CMI) et fongicide (CMF) des extraits aqueux et éthanolique a donné les résultats consignés dans le Tableau 3. Les colonies de Fusarium sp. et Phytophthora sp. ont eu des sensibilités différentes vis-à-vis des extraits aqueux et éthanolique de Mallotus oppositifolius. L'EE a fourni les meilleurs résultats de CMI, avec 25 $\mathrm{mg} / \mathrm{mL}$ sur Fusarium sp. et $50 \mathrm{mg} / \mathrm{mL}$ sur Phytophthora sp. contre $100 \mathrm{mg} / \mathrm{mL}$ sur les deux phytopathogènes pour l'ETA.

Tableau 3: Concentration minimale inhibitrice (CMI) et fongicide (CMF) des extraits aqueux et éthanolique de Mallotus oppositifolius sur Fusarium sp. et Phytophthora sp.

\begin{tabular}{l|l|l|l}
\hline Phytopathogène & Extrait végétal & CMI $(\mathbf{m g} / \mathbf{m L})$ & CMF $\mathbf{( m g} / \mathbf{m L})$ \\
\hline \multirow{2}{*}{ Fusarium sp. } & Extrait aqueux & 100 & 200 \\
\cline { 2 - 4 } & Extrait éthanolique & 25 & 200 \\
\hline \multirow{2}{*}{ Phytophthora sp. } & Extrait aqueux & 100 & 200 \\
\cline { 2 - 4 } & Extrait éthanolique & 50 & 200 \\
\hline
\end{tabular}

\section{$5 \quad$ DISCUSSION}

Le rendement des extraits aqueux et hydroéthanolique $70 \%$ ont été respectivement de $26,35( \pm 0,75 \%)$ et $34,47( \pm 1,25 \%)$. L'extrait hydro-éthanolique a donné un meilleur rendement par rapport à celui de l'extrait total aqueux. En effet, l'extraction consiste en un 
transfert de masse des solutés (poudre) vers le solvant. Le solvant est donc l'un des paramètres influents sur la nature et la cinétique de ce transfert de masse (Anonyme, 2019). Dès lors, le rendement de l'extraction sera d'autant plus élevé que les composés à extraire seront solubles dans le solvant d'extraction. L'éthanol $70 \%$ a certainement permis d'extraire à la fois les molécules solubles dans l'alcool et les petites molécules polaires solubles dans l'eau, ce qui justifie son meilleur rendement. Les analyses phytochimiques réalisées au cours de cette étude ont montré que l'extrait $\mathrm{EE}_{14}$ renferme des polyphénols, des alcaloïdes, des flavonoïdes, des polyterpènes et stérols, des tanins galliques et des catéchines, des saponines et des quinones. L'extrait ETA $_{14}$ contient les mêmes composés mais ne contient pas de quinones Les métabolites secondaires contenus dans les extraits aqueux et éthanoliques peuvent influencer différemment la croissance mycélienne des isolats des deux champignons à cause de leur différence de composition qualitative et/ou quantitative. Plusieurs travaux soutiennent que la majorité des métabolites secondaires des plantes sont à la base des activités physiologiques contre les microorganismes pathogènes (Adeleye et al., 2008 ; Mambe et al., 2016; Orsot et al., 2016; Owhe-Ureghe et Akpo, 2016). L'étude de l'influence des différents extraits (total aqueux et éthanolique $70 \%$ ) sur la croissance mycélienne des isolats de Phytophthora sp. et Fusarium sp. a montré des propriétés antifongiques de Mallotus oppositifolius. Une réduction de la croissance mycélienne a été constatée parallèlement à l'augmentation de la concentration de l'extrait. Cet effet a été plus important avec l'extrait éthanolique $\left(\mathrm{EE}_{14}\right)$ pour lequel une inhibition de la croissance du mycélium de $50 \%$ a été obtenue à la dose de 6,5 $\mathrm{mg} / \mathrm{mL}$ sur Fusarium sp. et $12 \mathrm{mg} / \mathrm{mL}$ sur Phytophthora sp. contre respectivement 24 $\mathrm{mg} / \mathrm{mL}$ et $30 \mathrm{mg} / \mathrm{mL}$ avec l'extrait aqueux $\left(\mathrm{ETA}_{14}\right)$. L'analyse de la variance à un facteur montre que la différence entre les concentrations est significative pour chaque isolat fongique au seuil $P<0,0001$ selon le test LSD de Fisher. Les taux d'inhibitions obtenus avec l'extrait éthanolique sur Fusarium sp. sont significativement supérieurs à ceux obtenus sur Phytophthora sp. pour les concentrations $\mathrm{C} \leq 12,5$ $\mathrm{mg} / \mathrm{mL}$. Ces deux souches fongiques testées ont eu des sensibilités différentes vis à vis des extraits aqueux et éthanolique. Nous pouvons affirmer que les colonies de Fusarium sp. ont été plus sensibles à l'extrait éthanolique $70 \%$ que les colonies de Phytophthora sp. Le rapport des $\mathrm{CI}_{50}$ donne $\mathrm{CI}_{50}\left(\mathrm{ETA}_{14}\right) / \mathrm{CI}_{50}\left(\mathrm{EE}_{14}\right)=24 / 7=$ 3,43 pour Fusarium sp. et $\mathrm{CI}_{50}\left(\mathrm{ETA}_{14}\right) / \mathrm{CI}_{50}$ $\left(\mathrm{EE}_{14}\right)=30 / 11=2,73$ pour Phytophthora sp. Cela signifie que l'EE $E_{14}$ est environ 3,43 fois plus actif sur Fusarium sp. que l'ETA 14 et 2,73 fois plus actif sur Phytophthora sp. que l'ETA I $_{14}$. Cela s'expliquerait par le fait que les principes actifs contenus dans l'extrait éthanolique ont un plus grand potentiel antifongique. Nous en déduisons que l'éthanol permet d'extraire mieux que l'eau, les métabolites secondaires de Mallotus oppositifolius auxquels les phytopathogènes étudiés sont sensibles. L'activité antifongique de l'extrait éthanolique $\mathrm{EE}_{14}$ pourrait aussi être due à une synergie d'action entre des métabolites extractibles dans l'eau et d'autres extractibles dans l'éthanol (Kouadio, 2013). Le pouvoir fongicide des feuilles de Mallotus oppositifolius a été le même $(\mathrm{CMF}=200 \mathrm{mg} / \mathrm{mL}$ ) aussi bien sur Fusarium sp. que sur Phytophthora sp. avec l'extrait aqueux, mais aussi avec l'extrait éthanolique. L'extrait $\mathrm{EE}_{14}$, ayant les plus faibles CMI $(25 \mathrm{mg} / \mathrm{mL}$ sur Fusarium sp. et $50 \mathrm{mg} / \mathrm{mL}$ sur Phytophthora sp.), est le plus actif sur l'inhibition de la croissance de Fusarium sp. et Phytophthora sp. Ces résultats corroborent ceux de Orsot et al. (2015) tout en restant plus faibles. En effet, ces auteurs ont montré l'activité antifongique de Mallotus oppositifolius sur Sclerotium rolfsii avec une CMI de $100 \mathrm{mg} / \mathrm{mL}$ et une CMF de $200 \mathrm{mg} / \mathrm{mL}$ avec l'extrait aqueux et une CMI de $12,5 \mathrm{mg} / \mathrm{mL}$ et une $\mathrm{CMF}$ de $25 \mathrm{mg} / \mathrm{mL}$ avec l'extrait éthanolique. Cette différence pourrait être due aux caractéristiques des champignons testés ou aux méthodes d'extraction des extraits 
éthanoliques. Selon la classification de Kra (2016), les CMF de nos différents extraits sont tous supérieurs à $50 \mathrm{mg} / \mathrm{mL}$, leur activité est donc très faible. Cette activité pourrait être améliorée en purifiant nos extraits par un fractionnement bio-guidé. La comparaison de nos résultats avec ceux des travaux antérieurs réalisés sur les mêmes souches fongiques par Doga et al. (2017) montre que les extraits

\section{CONCLUSION}

L'étude de l'activité antifongique de Mallotus oppositifolius a révélé des effets inhibiteurs dosedépendants aussi bien sur les colonies de Phytophthora sp. que celles de Fusarium sp. L'extrait éthanolique de Mallotus oppositifolius a donné une $\mathrm{CI}_{50}$ de $6,5 \mathrm{mg} / \mathrm{mL}$ sur Fusarium sp. et de $12 \mathrm{mg} / \mathrm{mL}$ sur Phytophthora sp. par contre l'extrait aqueux a permis d'obtenir une $\mathrm{CI}_{50}$ de $24 \mathrm{mg} / \mathrm{mL}$ et une $\mathrm{CI}_{50}$ de $30 \mathrm{mg} / \mathrm{mL}$ respectivement sur Fusarium sp. et Phytophthora $s p$. La CMI de l'extrait éthanolique de Mallotus oppositifolius a été de $25 \mathrm{mg} / \mathrm{mL}$ sur Fusarium sp. et de $50 \mathrm{mg} / \mathrm{mL}$ sur Phytophthora sp. contre 100 $\mathrm{mg} / \mathrm{mL}$ sur les isolats de Fusarium sp. et

\section{REMERCIEMENTS}

Nos remerciements vont d'abord au Centre National de Floristique (CNF) de l'Université Félix Houphouët-Boigny de Cocody-Abidjan, pour la confirmation des noms des espèces végétales identifiées et recensées. Ensuite, nous exprimons notre gratitude aux responsables des Laboratoires du Département Agriculture et Ressources Animales de l'Institut National

\section{RÉFÉRENCES}

Ackah JA: 2004. Spectre anti infectieux de MISCA- $\mathrm{F}_{3}$ sur la croissance in vitro de $C$. albicans, T. mentagrophytes, T. rubrum, $C$. neoformans, $A$. fumigatus et $A$. flavus. Mémoire de D.E.A de Biotechnologie option Pharmacologie. UFR Biosciences, Université de CocodyAbidjan, Côte d'Ivoire, 34 p.

Adeleye A, Ezekiel O, Smith S, Odusola O. et Sobande J: 2008. Antibacterial activity aqueux de Mallotus oppositifolius ont une activité antifongique plus faible que l'extrait brut aqueux de Crotalaria retusa L. qui a donné une CMF de $6,25 \mathrm{mg} / \mathrm{mL}$ sur Phytophthora sp. et une CMF de $12,50 \mathrm{mg} / \mathrm{mL}$ sur Fusarium sp. Nos extraits $\mathrm{ETA}_{14}$ et $\mathrm{EE}_{14}$ sont plutôt fongistatiques que fongicides pour les concentrations comprises entre $25 \mathrm{mg} / \mathrm{mL}$ et $100 \mathrm{mg} / \mathrm{mL}$.

Phytophthora sp. pour l'extrait aqueux. Le meilleur solvant a été l'éthanol qui permet une meilleure extraction des métabolites secondaires de $M$. oppositifolius actifs sur les phytopathogènes testés. Le pouvoir inhibiteur de l'extrait éthanolique a été plus important sur la croissance mycélienne des isolats de Fusarium sp. que celle de Phytophthora sp. Ce travail pourra être poursuivi par des études de fractionnement des extraits éthanoliques de Mallotus oppositifolius suivi de tests in vivo qui permettront de déterminer les concentrations efficaces et suffisantes contre Fusarium sp. et Phytophthora sp.

Polytechnique Félix Houphouët-Boigny de Yamoussoukro qui ont bien voulu mettre à notre disposition les équipements nécessaires pour la réalisation de ce travail. Ce sont en particulier les responsables et le personnel du Laboratoire de Phytopathologie et de Biologie Végétale.

of extracts of Alchornea cordifolia (Schum and Thonn) Mull. Arg., Boerhavia diffusa (L) and Bridellia micrantha (Hoscht) Baill. used in traditional medicine in Nigeria on Helicobacter pylori and four diarrhoeagenic bacterial pathogen. African Journal of Biotechnology 7(20): 3761-3764. 
Anonyme:

2018.www.prota4u.org/database/protav 8.asp?h=M4\&t=Mallotus,oppositifolius

Anonyme: 2019. Extraction vegetate (le cœur de métier de Berkem,) http://www.berkem.com/fr/expertise/ extraction-vegetale

Brent KJ et Hollomon DW, 2007 : Fungicide resistance in crop pathogens: how can it be managed? Fungicide Resistance Action Committee (FRAC) Monograph No.1, CropLif International, 60p.

Coraf: 2010. Des extraits végétaux à la place des produits de synthèse, $\mathrm{N}^{\circ} 56$ juillet à septembre, $16 \mathrm{p}$.

Doga D, Zirihi GN. et Zézé A : 2017. Propriétés antifongiques des légumineuses médicinales de Côte d'Ivoire: cas de Crotalaria retusa L. (Fabaceae) sur la croissance in vitro de Phytophthora sp. et Fusarium solani, deux champignons phytopathogènes. European Scientific Journal, 13(3): 371-384.

Flood J : 2006. The threat from global spread of cocoa pests and diseases: hypothetical scenario or clear and present danger. In: proceedings of the 15th International Cocoa Research Conference, 9-10 October 2006. San Jose (Costa Rica), 857-872.

Hibar K, Medja D. et Mahjoub ME: 2007. Effect of some chemical and biological fungicides on mycelial growth and disease severity of Fusarium oxysporum $\mathrm{f}$ sp racidis lycopersici. Tropicultura, 25(3): 146-1452.

Kouadio NJ: 2013. Evaluation de l'activité antibactérienne de quatre (4) plantes médicinales sur la croissance in vitro de bactéries multi-résistantes. Mémoire de Master, Université Nangui Abrogoua, Abidjan, 53p.

Kouadio NJ, Guessennd NK, Kone MW, Moussa B, Koffi MZ, Guede KB, Yao K, Bakayoko A, Tra bi HF. et Dosso M: 2015. Evaluation de l'activité des feuilles de Mallotus oppositifolius (Geisel.) Müll.
Arg. (Euphorbiaceae) sur des bactéries multirésistantes et criblage phytochimique. International Journal of Biological and Chemical Sciences, 9(3): 12521252.

Kra AKM: 2016. Recherche bio guidée de composés antifongiques à partir des plantes médicinales de Côte d'Ivoire. Thèse de Doctorat de l'UFR Biosciences, Université Félix Houphouët Boigny, Abidjan, Côte d'Ivoire, $242 \mathrm{p}$.

Kra KD, Diallo HA, Kobenan K, Kone D.et Kouadio YJ : 2011. Diagnostic de la fusariose du bananier sur les cultivars grande naine (Musa AAA) et corne 1 (Musa AAB) à la périphérie du District d'Abidjan (Côte d'Ivoire). International Journal of Biological and Chemical Sciences 5(4): 1501-1514.

Lass R A: 1985. Disease. In Cocoa. Wood G.A.R. \& Lass R. A. Eds. 4th Edition, Longman, 265-365.

Leroux P. et Credet A : 1978. Document sur l'étude de l'activité des fongicides. INRA Versaille, France, 12 p.

Mambé FT, Voukeng IK, Beng VP. Et Kuete $\mathrm{V}$ : 2016. Antibacterial activities of methanol extracts from Alchornea cordifolia and four other Cameroonian plants against MDR phenotypes. Journal of Taib University, Medical Sciences, 11(2): 121-127.

Mangambu M, Mushagalusa K. et Kadima N: 2014. Contribution à l'étude phytochimique de quelques plantes médicinales antidiabétique de la ville Bukavu et ses environs (Sud-Kivu, R.D.Congo). Journal of Applied Biosciences, 75: 6211-6220.

Orsot BAMB, Soro S, Konkon NG, Kone D. et Zirihi GN: 2016. Étude ethnobotanique et évaluation in vitro de l'activité antifongique des extraits de l'écorce de Zanthoxylum gilletii sur deux souches phytopathogenes de Sclerotium 
rolfsii, Journal of Applied Biosciences 98: 9309 - 9322.

Orsot BAMB, Soro S, Ouattara D, N'guessan EK. et Zirihi GN: 2015. Étude ethnobotanique et évaluation in vitro de l'activité antifongique des extraits de feuille de Mallotus oppositifolius sur deux souches phytopathogènes de Sclerotium rolfsii, Europeen Scientific Journal 36 : $1857-$ 7881.

Owhe-Ureghe UB. et Akpo CO : 2016. In Vitro Antibacterial Activity of Mallotus oppositifolius and Costus afer Against Some Bacterial Isolates. UK Journal of Pharmacentical and Biosciences 4(3): 51-55.

Picanc OM, Leite GLD, Guedes RNC. et Silva EA: 1998. Yield loss in trellised tomato affected by insecticidal sprays and plant spacing. Crop Prot. 17: 447-452.

Soladoya MO, Ikotun T, Chukwuma EC, Ariwaodo JO, Ibhanesebor GA, AgboAdediran OA. et Owolabi SM : 2013. Our plants, our heritage: Preliminary survey of some medicinal plant species of South westem University Nigeria. Campus Ogun State. Nigeria Annals of Biological Research, 4(12): 27-34.

Soro D, Koné MW, Koné D. et K. Kamanzi : 2012. Evaluation de l'activite antifongique par bio-autographie de quelques plantes medicinales de côte d'ivoire contre deux formes speciales de Fusarium oxysporum. Agronomie Africaine 24(1): 19 - 28.

Zehharb G, Touhami AO, Badoc A. et Douira A: 2006. Effet des Fusarium des eaux de rizière sur la germination et la croissance des plantules de riz. Bulletin de la Société de Pharmacie de Bordeaux. 145: 718.

Zirihi GN, Kra AKM. et Guédé-Guina F: 2003. Evaluation de l'activité antifongique de Microglossa pyrifolia (Lamarck) O. Kuntze (Asteraceae) "PYMI" sur la croissance in vitro de Candida albicans. Revue de Médecine et de Pharmacopée Africaine, 17: 11-18. 